\title{
THREE-DIMENSIONAL STRESS PROBLEM OF A FINITE THICK PLATE WITH A THROUGH- CRACK UNDER TENSION
}

\author{
M. N. Bapu Rao \\ Structures Division. National Aeronaufical Laboralory, Bangalore 560017, India
}

ABSTRACT

The three dimensional state of stress in a finite thick rectangular plate with a through-crack under tension is investigated. It is found that the in-plane stresses and the transverse normal stress are singular while the transverse shear stresses are of the order of unity. The only type of singularity encountered is that of inverse square root all through the plate thickness including the corner points at the plate faces. The stress intensity factor which is found to vary with $z$ and the thickness ratio $\mathbf{h} / \mathbf{a}$ drops rapidly in a thin boundary layer near the plate faces, but without actually vanishing there. The stress intensity factor reduces exactly to that for the plane strain case when $\mathrm{h} / \mathrm{a} \rightarrow \boldsymbol{\infty}$. All the three components of the displacement field are finite at the crack front.

KEY WORDS

re; through-crack; stress intensity factor; thick plate; Eragtucity; three-dimensional analysis.

INTRODUCTION

Recent studies (Hartranft and Sih, 1969;Sih, Williams and Swedlow, 1966, Folias, 1975, 1980) in three dimensional (3-D) cracked-plate problems with cracks emerging at the free surfaces have raised some questions such as, (1) the type of singularities involved at the crack front, in particular, at the corner points where the crack front penetrates the free plate faces, (2) the type of variation of the stress intensity factor (SIF) across the plate thickness, in particular, near the plate faces, (3) the finiteness or otherwise of the 
displacement components at the crack front. The studies carried out in the above mentioned references point to the existence of the inverse square root singularity $1 /(\bar{r})^{1 / 2}$ in the stress field interior to the plate thickness with a predominantly plane strain type of deformation; no numerical results are prsented there. However, near the plate faces, while the first two references do not throw any light as to either the type of stress singularity or the deformation character, the studies in next two references reveal a Possion's ratio-dependent stress singularity, viz, $1 /(\bar{r})^{1 / 2}+2 S^{m}$ indicating a displacement singularity at the crack front for $\mathrm{M}>1 / 4$; in contrast, in the first two references the displacement finiteness condition at the crack front is enforced on the solution. The results of (Benthem, 1976; Kawai, 1975) for the stresses near the corner point of a quarter plane crack exhibit $1 /(\bar{r})^{\infty}$ singularity with $\alpha$ varying from 0 to $1 / 2$ in the former's case and $1 / 2$ to 1 in latter's investigation. The experimental studies of reference (Villarreal, Sih and Hartranft, 1975) serve to confirm the qualitative results of (Hartranft, 1969; Sih, 1966; Folias, 1975,1980) regarding the character of the singular deformation field interior to the plate thickness; but, near the plate faces, a rapid decrease in SIF values is indicated, suggesting a reduction in the strength of singularity in the region.

In this paper, the 3-dimensional state of stress in a thick rectangular plate with a through-crack under tension is investigated. The mathematical formulation of the problem is based on the 3-dimensional elasticity equations, (Lure, 1964). The solution obtained satisfies, exactly, the stress-free conditions at the crack surfaces as also the plate faces; and the boundary conditions (B.C) at the exterior edges of the plate, including those of the applied tensile loading, are satisfied in the least square sense. It is found that the inplane stresses and the transverse normal stress are singular at the crack front while the transverse shear stresses are of the order of unity.

During the early stages of the formulation of the present problem some interesting features concerning the singular stress field at the crack front were observed. Some of these results were reported in (Bapu Rao, 1981). Subsequently, further formulation and numerical studies were carried out. In this paper,only essential features of the formulation of the problem and numerical results for two typical problems of a thick plate with a through-crack under tension are presented. These results and their comparison with existing results lead to some important conclusions.

\section{FORMULATION OF THE PROBLEM}

In view of the cylindrical coordinate system chosen $(\bar{r}, \bar{\theta}, \bar{z})$ in association with the Cartesian coordinate system ( $\bar{x}, \bar{y}, \bar{z})$, See Fig. 1, it is necessary to consider for analysis only the 
region defined by $(-A) \leqslant \vec{x} \leqslant(L-a),(-B) \leqslant \vec{y} \leqslant B$ and $(-h) \leqslant 2 \leqslant h$, with appropriate continuity conditions at $\vec{x}=-\mathbf{a}$ and boundary conditions at other edges, which are given as

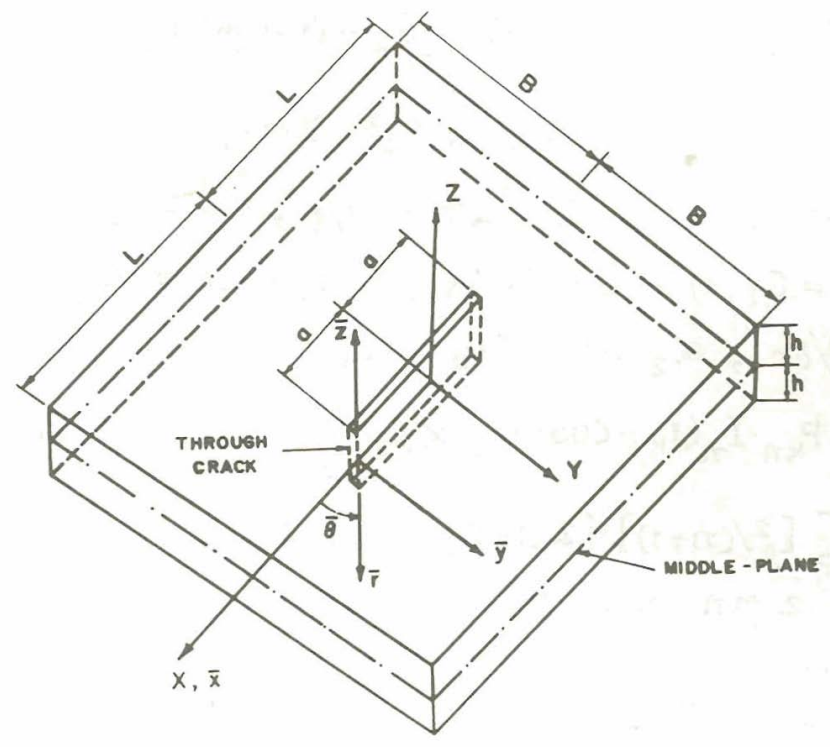

Fig. 1. Coordinate system and plate dimensions

at $\vec{x}=-a, u=\sigma_{x z}=\sigma_{x y}=0$; at $\bar{x}=(L-a), \sigma_{x}=\sigma_{x y}=\sigma_{x z}=0$

at $\vec{y}= \pm B, \quad \sigma_{y}=\sigma$ (applied tension), $\sigma_{y z}=\sigma_{x}^{x y} \equiv 0$

The governing equations (G.E) of the problem are the 3-D elasticity equations in terms of displacement components $(u, v, w)$. These equations as also those for the stress components $\left(\sigma_{r}, \sigma_{\theta}, \sigma_{r \theta}, \sigma_{z}, \sigma_{r_{z}}, \sigma_{\theta z}\right)$ are available in (Hartranft, 1969; Lure, 1964). It is convenient to nondimensionalize $(\bar{r}, \bar{\theta}, \bar{z})$ with respect to $a: r=\bar{r} / a, \theta=\bar{\theta}$, and $z=z / a$ where $2 a$ is the crack length. The solution to G.E must satisfy the following boundary conditions =

(i) at the plate faces $(z= \pm \mathrm{h} / \boldsymbol{a}= \pm \boldsymbol{\jmath}), \sigma_{r_{z}}=\sigma_{\theta z}=\sigma_{z}=0$;

(ii)at the crack surfaces $(\theta= \pm \pi), \sigma_{r_{\theta}}=\sigma_{\theta}=\sigma_{\theta z}=0$

and also those defined by eqs. I and 2. The solution to G.E is given as

$$
u_{i}=\operatorname{Re} \sum_{k=1}^{\infty} \Psi_{i}\left(z, \lambda_{k}\right) \cdot \alpha_{i} x_{a}\left(r, \theta, \lambda_{k}\right)+x_{i}(r, \theta)+
$$




$$
\begin{aligned}
& +\sum_{l=1}^{\infty} F_{i}(z) \cdot \alpha_{i} X_{b}(r, \theta, l) ; i=1-3 \\
& u_{1}=u / a, u_{2}=v / a, \quad u_{3}=w / a \\
& \Psi_{1}=\Psi_{2}=\left[-c_{k} \cdot \bar{C}_{k}-(z / \rho) \cdot s_{k} \cdot \bar{S}_{k}+(1-2 \xi n) \cdot c_{k} \cdot \bar{S}_{k} / \lambda_{k}\right] \\
& \Psi_{3}=\left[S_{k} \cdot \bar{C}_{k} \cdot \lambda_{k} / \rho-C_{k} \cdot \bar{S}_{k} \cdot \lambda_{k} \cdot z / \rho^{2}+2(1-s n) \cdot s_{k} \cdot \bar{S}_{k} / \rho\right] \\
& C_{k}=\cos \lambda_{k} \cdot z / \rho, S_{k}=\sin \lambda_{k} \cdot z / \rho, F_{3}=-S_{l}(z) \cdot l \pi / \rho \\
& F_{1}=F_{2}=C_{l}(z)=\cos l \pi z / \rho, S_{l}(z)=\sin l \pi z / \rho, X_{3}=0 \\
& \alpha_{1}=\partial / \partial r, \alpha_{2}=1 / r \cdot \partial / \partial \theta, \alpha_{3}=1 ; r_{k}=\lambda_{k} \cdot r / s \\
& X_{a}=\sum_{n} P_{k n} \cdot I_{n}\left(r_{k}\right) \cdot \cos n \theta, X_{b}=\sum_{n} t_{\ln } \cdot I_{n}(l \pi r / s) \cdot \cos n \theta \\
& X_{1}=-\sum_{n}[2 /(n+1)] \cdot[2(1-2 \mu)-n] \cdot A_{n} \cdot r^{n+1} \cos n \theta \\
& -2 \mathrm{~s} n \cdot \mathrm{N}_{n} \cdot r^{n-1} \cdot \cos n \theta \\
& X_{2}=-\sum_{n}[2 /(n+1)] \cdot[4(1-n)+n] \cdot A_{n} \cdot r^{n+1} \sin n \theta \\
& +2 m n \cdot N_{n} \cdot r^{n-1} \cdot \sin n \theta
\end{aligned}
$$

$i 1$ eưs. 3-10, $I_{\boldsymbol{n}}\left(\boldsymbol{\lambda}_{\boldsymbol{k}}, \boldsymbol{r} / \boldsymbol{\jmath}\right)$ and $I_{\boldsymbol{n}}(\mathbf{I} \boldsymbol{\pi} \cdot \boldsymbol{r} / \boldsymbol{\xi})$ are modified Bessel functions of the first kind, and $\lambda_{k}$ are the complex roots of the equation Sin $2 \lambda_{x}+2 \lambda_{x}=0$, (this equation is obtained by satisfying the stress free B.C's at plate faces). Here $\bar{C}_{k}$ and

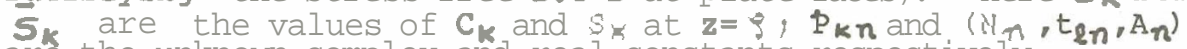
are the unknown complex and real constants respectively.

The first terms in eq. 3 automatically satisfies the B.C. at the plate faces by virtue of the equation $\sin 2 \lambda_{k}+2 \lambda_{k}=0$, ( $k=1,2,3, \ldots)$ ); these conditions are also satisfied independentiy by the second and third terms of eq. 3 when considered together, leading to the elimination of some of the associated unknown constants. In order to satisfy the B.C at the crack surfaces (these B.C.'s are to be satisfied by all the three terms in eq. 3 considered together) it is useful to express the stresses in the form of a power series in $\boldsymbol{r}$, Sine and Cosine functions of $\partial$, and Fourier Series in $z$. This can be accomplished by expressing $\left(C_{k}, S_{k} z / \rho\right)$ and $\left(S_{k}, C_{k} z / \rho\right)$ in eq. 5 in Fourier series of the form $C_{l}(z)$ and $S_{l}(z), \quad l=1,2,3, \ldots$, as also terms of the order of unity $O\left(z^{0}\right)$, by taking advantage of the power series form in $r$ of Bessel functions and biharmonic functions, and by virtue of the existing Sine and Cosine terms in $\theta$ present in eqs. 7-10. It is now appropriate 
to consider the range of $\mathrm{n}$ occurring in the expressions for the stresses and displacements, eqs. 7-10. The range of $\mathrm{n}$ is determined from the satisfaction of the finiteness condition of displacement components at the crack front. It is found that there are four groups of $n$ whose ranges are: $n=2 j \pm 1 / 2, n=2 j+1$ and $n=2 j, \quad j=0,1,2, \ldots$. significantly the limit placed is on the lower value of $n,(n=-1 / 2)$. Satisfaction of the B.C.'s at the crack surfaces (by setting $\theta= \pm \pi$ in the expressions for $\sigma_{\boldsymbol{\theta}}$, $\sigma_{\theta z}$, and $\left.\sigma_{r_{\theta}}\right)$ with respect to each power of $r$ for each range of $n$ lead to singular stresses. This method of satisfying B.C's has been employed for a two dimensional bending problem of a cracked plate, (Murthy, 1981). The B.C"s at the exterior edges of the plate as defined by eqs. 1 and 2 are satisfied with respect to stress resultants (rather than unit stresses) and average u over the thickness, in the least square sense. The satisfaction of all B.C.'s mentioned above lead to the determination of all unknown constants for given plate dimensions and h/a ratios.

\section{RESULTS AND DISCUSSIONS}

It is found that the in-plane stresses $\left(\sigma_{\boldsymbol{r}}, \sigma_{r}, \sigma_{\theta}\right)$ and $\sigma_{z}$ are singular with a $1 /\langle\bar{r})^{\frac{1}{2}}$ singularity; the stresses $\sigma_{r z}$ and $\sigma_{\theta z}$ are of the order of unity. The corresponding expressions are givenas

$$
\begin{aligned}
& \sigma_{r}=K(z) \cdot\left[1 / 4(2 \bar{r})^{1 / 2}\right] \cdot(5 \cos \theta / 2-\cos 3 \theta / 2)+O\left(\bar{r}^{0}\right) \\
& \sigma_{\theta}=K(z) \cdot\left[1 / 4(2 \bar{r})^{1 / 2}\right] \cdot(3 \cos \theta / 2+\cos 3 \theta / 2)+O\left(\bar{r}^{0}\right) \\
& \sigma_{r \theta}=K(z) \cdot\left[1 / 4(2 \bar{r})^{1 / 2}\right] \cdot(\sin \theta / 2+\sin 3 \theta / 2)+O\left(\bar{r}^{0}\right) \\
& \sigma_{z}=K^{*}(z) \cdot\left[4 m / 2(2 \bar{r})^{1 / 2}\right] \cdot \cos \theta / 2+O\left(\bar{r}^{0}\right) \\
& \sigma_{r z}=O\left(\bar{r}^{0}\right), \quad \sigma_{\theta z}=O\left(\bar{r}^{0}\right)
\end{aligned}
$$

In egs. 11 and $12, \boldsymbol{g}(\mathbf{z})$ is the stress intensity factor and $\mathbf{K}(\mathbf{z})$ is the factor associated with the singular term of $\sigma_{\mathbf{z}}$ " The corresponding expressions are given as

$$
\begin{aligned}
K(z)= & -2(2 a)^{1 / 2} \cdot G\left[\left\{\operatorname{Re} \sum_{k=1}^{\infty}\left(m / \rho^{2}\right) \cdot P_{k(-1 / 2)}+4 A_{-1 / 2}\right\}+\right. \\
& \left(1 / \rho^{2}\right) \cdot \sum_{l=1}^{\infty} 2(-1)^{l} \cdot C_{l}(z) \times\left\{\operatorname{Re} \sum_{k=1}^{\infty} \lambda_{k}^{2} \cdot P_{k(-1 / 2)} \times\right. \\
& \left.\left.\left(m \cdot A_{k l}-(1+\rho) \cdot G_{k l}\right)-(-1)^{l} \cdot(l \pi / 2)^{2} \cdot t_{l(-1 / 2)}\right\}\right]
\end{aligned}
$$




$$
\begin{aligned}
K(z)= & -2(2 a)^{1 / 2} \cdot G\left[\left\{R e \sum_{k=1}^{\infty}\left(1 / \Omega n \rho^{2}\right) \cdot P_{k(-1 / 2)}+4 A_{-1 / 2}\right\}+\right. \\
& \sum_{l=1}^{\infty}(-1)^{l} \cdot\left(2 / m \rho^{2}\right) \cdot C_{l}(z) \times\left\{R e \sum_{k=1}^{\infty} \lambda_{k}^{2} \cdot A_{k l} \cdot P_{k(-1 / 2)}+\right. \\
& \left.\left.(-1)^{l} \cdot(l \pi / 2)^{2} \cdot t_{l(-1 / 2)}\right\}\right]
\end{aligned}
$$

Xn egs. 13 and $14, p_{k(-1 / 2)}{ }^{A}-1 / 2$ and $t_{\ell(-1 / 2)}$ are the unknown constants referred to earlier with respect to $n=-1 / 2$. Here, $A_{k \ell}$ and $G$ ke are known functions of $\lambda_{k}$ and $1 \pi ; \quad G$ is the shear modulus it can be observed from eqs. 11 that the only type of singugh the encountered is that of inverse square root all there the crack plate thickness including the corner points whe (Folias, 1975) a

Polston"s ratio-dependant $1 /(\bar{r})^{1 / 2+2 \Omega^{n}}$ type of singularity was exhlbited, in the present analysis, It is noted, no such dependence on $M \pm$ inoosed, the angular variation of slngular stresses are identical to those of (Hartranft, 1969). The SIF is found to depend $O \mathrm{z}$ and $\mathrm{h} / \mathrm{a}$ ratio. It can be nated Eron 53 that it does not vanish at the plate faces and, therefore,the ln-plane stresses preserve thelr $1 /(\bar{I})^{1 / 2}$ alngularity all though the plats thickness including the corner ofinta at the plate faces. It should also be observed Erom eq. Is that $\mathrm{K}(\mathrm{z})$ ranishes at the plate faces, thereby leading to tanshing of $\sigma_{z}$ here. It is interesting to note that the expressions for $\boldsymbol{x}(\boldsymbol{z})$ and $(\mathrm{h})$ reduce exactly to those for the plane strain case when $2 h \rightarrow \infty,(\rho \rightarrow \infty)$; thus olave straln results aare recovered fron eos. 11 and 12 . It is noteworthy that all the three displacenent components are finfte ah the crack front. In contrast, in (Folias, 1975), ther were found to be singular for certain values of $m(n>1 / 4)$ in the viclnity of the corner polnts.

The preceding dscussion was nade without any reference to numerleal results but, solely from the nature of the mathematical expresslons presented In eqs. 11-14 and also those not presented here to save space. Therefore, it will be Interesting to discuss the numetidel results obtained. Pig, 2 shows the distribution of sts acrosis the plate thickness for the dese wth $\mathrm{p} / \mathrm{t}=0.875, \quad \mathrm{a} / \mathrm{L}=0.5$ and $\mathrm{h} / \mathrm{\alpha}=\mathrm{s}=1.5$ and for applied tensile 1oading. Corresponding results obtained by finite element retrod, (FEM), (Raju and Nerman, 1977) and the boundary integral equations method, (BIE), (Tan and Fenner, 19791 are also presented for comparison purposes. The $\mathbf{x}$ alues from the plate middle plane $z=0$ to about $z=0.6 \rho$

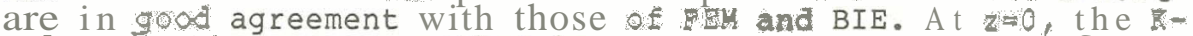
value of the present method is about 1.438 while that of MRE 18 1.401; $1 t$ is of interest to note that the corresponding plane strain value is equal to about 1.43. In fact, s can be 


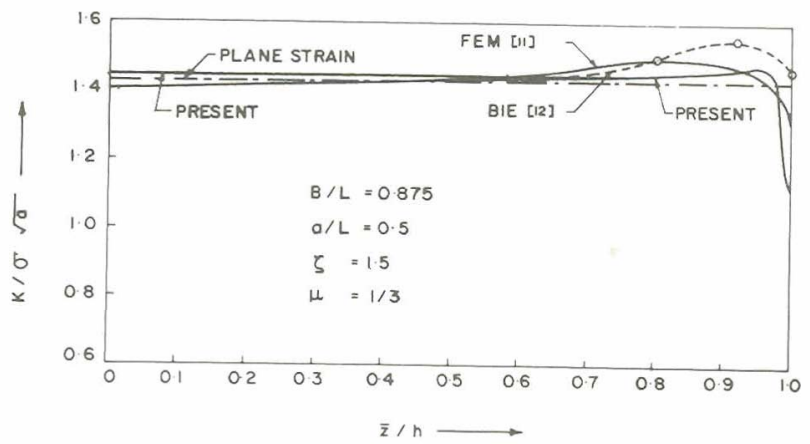

Fig. 2. SIF distribution across plate thickness

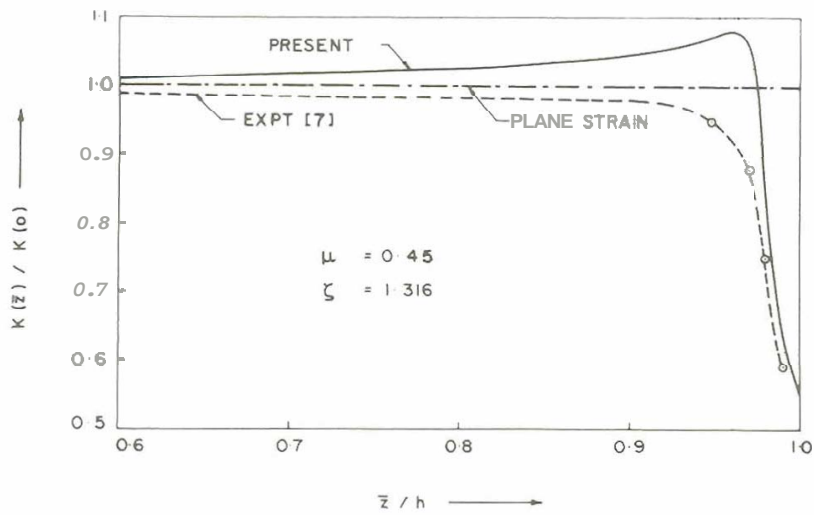

Fig. 3. SIF distribution across plate thickness (normalized by mid-plane value)

observed from Fig. 2, the singular deformation character is more or less equal to that of plane strain in the region interior to the plate thickness $\mathbf{z}=0.0-0.6 \rho ;$ this is in agreement with the qualitative behaviour predicted in (Hartranft, 1969; Sih, 1966; Folias, 1975) for this zone. However, beyond this zone, the $\mathrm{K}$-value increases gradually with increase in $\mathrm{z}$ upto a maximum value and then drops rapidly in a small boundary layer near the plate faces. The location and magnitude of the maximum value of the present method are different from those of (Raju, 1977; Tan, 1979). Also, the Kvalue at $\mathbf{z}=\mathrm{g}$ (free plate faces) is much less than those of these references; when compared to the mid-plane value the 
percentage drop is about 19.86 for the present case. In this connection it should be pointed out that the FBM can not represent accurately drastic changes. In slopes such as those realized in present $\mathrm{K}$-distribution in the boundary layer region. However, the slopes encountered at points $z=9$ and at $z=3$ for the FEM case (see Pig. 2) do suggest a trend which leads to a larger drop than numerically indicated. Pig. 3 shows a comparison of the SIP distributions across the thickness normalised by the $\mathrm{K}(0)$ value (mid-plane value) for the case with $\mathcal{S}=1.316$ obtained in this paper and by the photoelastic method (V1llarreal, 1975). As can be observed from this figure, the agreement between the two distributions is reasonabliy good for the region, $z=(0.0-0.85) \rho$ (approximately); the predominantly plane strain type of behaviour observed here confirms the qualitative results of (Hartranft, 1969; Sih, 1966; Polias, 1975) for this region. Although for larger values of $z$ increasing differences are observed, the significant feature noticed here lies in the qualitatively identical distributions of the two methods, all through the thickness except in a small region $z=0.9$ to $0.97 \mathrm{~s}$ approximately. At the plate faces, the percentage reduction in the SIF value is found to be 45 as against 40 (approximately) of (villerreal, 1975).

Based on the nature of the mathematical description of the singular field and the physical character of the numerically evaluated SIF distribution, V fified by experimental results, it can be concluded that $l /(\bar{r})$ His the only type of singularity present all through the plate thickness, although the strength of singularity decreases near the plate faces. Secondly, the region interior to the plate thickness experiences a more or less plane strain type of singular deformation; but, near the plate faces, there is a clear departure from this behaviour as suggested by the rapid decrease in SIF values. The displacements are finite at the crack front all through the plate thickness.

\section{REFERENCES}

Bapu Rao,M.N.(198I). Int.J.Frac.,17, R 43-46.

Benthem,J.P. and W.T.Ro1ter(1976). J.App.Mech.43, 374-384.

Pollas, E.S.(1975). JoApp.Mechen 42, 663-674.

Fol1as, E.S. (1980). Int.J.Frac.,16, 335-348.

Hartranft, R.J., and G.C.Sih(1969) = J.Math. and Mech. 1, , 123-138.

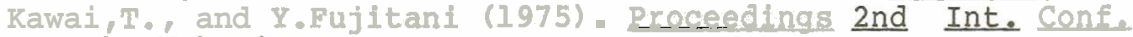
Meche Behaviour of Mat. Boston.

Iure, A.I.(1964). Three Dimensional Problems of the Theory of Elasticitz- Interscience publishers, New York.

Murthy,M.V.V., R.N.Raju, and S.Viswanath (1981). Intojorac-. $17,537-552$.

Raju,I.S., and J.C.Newman, JI (1977). NASA TN P-8414 Sih,G.C., M.L.WIIIIams, and J.L.Swedlow(I966). AFMT $=$ TR_66_242 Tan, C.L., and R.T.Eenner (1979). Proc. Re Soc. (London), A369, 243-260.

Viliarreal,G., G.C.S1h, and R.J.Bartianft (1975). J.App.Mech.. 42, $9-14$. 